Benedetto Bongiorno, Luisa Di Piazza*, Department of Mathematics, University of Palermo, Via Archirafi 34, 90123 Palermo, Italy

Valentin Skvortsov $\dagger^{\dagger}$ Department of Mathematics, Moscow State University, Moscow 119899, Russia

\title{
A NEW FULL DESCRIPTIVE CHARACTERIZATION OF DENJOY-PERRON INTEGRAL
}

\begin{abstract}
It is proved that the absolute continuity of the variational measure generated by an additive interval function $F$ implies the differentiability almost everywhere of the function $F$ and gives a full descriptive characterization of the Denjoy-Perron integral.
\end{abstract}

\section{Introduction}

It is well known (see for example [8]) that the variational measure generated by the indefinite Henstock-Kurzweil integral ( $H K$-integral) of any $H K$-integrable function is absolutely continuous with respect to Lebesgue measure. This property which is equivalent to the Strong Lusin Condition (SLC) introduced in [6] was used by several authors to give a so called (For this terminology see [8, pages 93 and 115].) partial descriptive characterization of the HenstockKurzweil integral. Namely it was proved (see [2], [7], [8]) that if an additive interval function $F$ is differentiable almost everywhere and generates an absolutely continuous variational measure (or, in other words, satisfies SLC), then it is the indefinite $H K$-integral of $F^{\prime}$.

We show here (Theorem 3 ) that the above property gives in fact a new full descriptive characterization of the $H K$-integral. In view of the above mentioned partial descriptive characterization it is enough to this end to prove that

\footnotetext{
Key Words: Henstock-Kurzweil integral, Denjoy-Perron integral, variational measure

Mathematical Reviews subject classification: Primary: 26A39, 26A42, 26A45

Received by the editors November 22, 1995

* Supported by M.U.R.S.T. of Italy.

†Supported by C.N.R. of Italy.
} 
the absolute continuity of a variational measure implies the differentiability almost everywhere of the generating function. This is done here in Theorem 1 and 2 below (see also Corollary 1 ). As the $H K$-integral is known (see [1], [3], [4], [5]) to be equivalent to the Denjoy-Perron integral, we conclude in Theorem 4 that the new characterization is equivalent to the descriptive definition of the latter integral in terms of $A C G_{*}$-functions. In the last section of this paper we extend our results to the variational measure defined by nonnegative gauges instead of positive functions $\delta$. This in particular gives an answer in a special one-dimensional case to a question of W. Pfeffer ([9]) on $\sigma$-finiteness of an absolutely continuous variational measure (see Theorem 5 below).

\section{Preliminaries}

We introduce some notation. If $E \subset \mathbb{R}$, then $|E|$ and $E^{0}$ denote respectively the Lebesgue measure and the interior of $E$. "Almost everywhere" (a.e.) is always used in the sense of the Lebesgue measure. An interval is always a compact subinterval in $\mathbb{R}$. A collection of intervals is called nonoverlapping whenever their interiors are disjoint. In this paper $[a, b]$ is a fixed interval of $\mathbb{R}$.

A partition $P$ in $[a, b]$ is a collection (possibly empty) $\left\{\left(I_{1}, x_{1}\right), \ldots,\left(I_{p}, x_{p}\right)\right\}$ where $I_{1}, \ldots, I_{p}$ are nonoverlapping subintervals of $[a, b]$ and $x_{j} \in I_{j}, j=$ $1, \ldots, p$. A partition $P=\left\{\left(I_{1}, x_{1}\right), \ldots,\left(I_{p}, x_{p}\right)\right\}$ is called anchored in $E \subset$ $[a, b]$ if $x_{j} \in E$ for each $j=1, \ldots, p$. For a given positive function $\delta$ on a set $E \subset A$, a partition $P=\left\{\left(I_{1}, x_{1}\right), \ldots,\left(I_{p}, x_{p}\right)\right\}$ in $[a, b]$ is called $\delta$-fine if $I_{j} \subset\left[x_{j}-\delta\left(x_{j}\right), x_{j}+\delta\left(x_{j}\right)\right]$ for each $j=1, \ldots, p$. We denote the family of all $\delta$-fine and anchored in $E$ partitions by $\mathcal{P}(\delta, E)$.

In this paper we denote by $F$ an additive interval function defined on the family of all subintervals of $[a, b]$. We call $F$ continuous if $F(I)$ tends to 0 when $|I|$ does. We can associate with the interval function $F$ a point function $\Phi(x)=F([a, x]), x \in[a, b]$ which is called the distribution function of $F$. Then for each interval $I=[c, d] \subset[a, b]$ we have

$$
F(I)=\Phi(d)-\Phi(c)
$$

Conversely if a point function $\Phi$ defined on $[a, b]$ is given, then (1) defines an associated interval function $F$. Note that in this case $F$ is continuous if and only if its distribution function $\Phi$ is continuous as a point function on $[a, b]$.

For an additive interval function $F$, a given positive function $\delta$ and a set $E \subset A$ we set

$$
\operatorname{Var}(F, \delta, E)=\sup \sum_{j}\left|F\left(I_{j}\right)\right|
$$


where "sup" is taken over all $P \in \mathcal{P}(\delta, E)$. Then we define

$$
\mathrm{V}_{F}(E)=\inf \operatorname{Var}(F, \delta, E),
$$

where "inf" is taken over all families of $\mathcal{P}(\delta, E)$, i.e. over all positive functions $\delta$ defined on $E$. We call $\mathrm{V}_{F}$ the variational measure generated by $F$ (or by $\Phi$ if $F$ is defined by (1)). Note that $\mathrm{V}_{F}$ is known to be a metric measure in $[a, b]$ (see [11]).

Definition 1 A variational measure $\mathrm{V}_{F}$ is called absolutely continuous on a set $E \subset[a, b]$ if $|N|=0$ implies $\mathrm{V}_{F}(N)=0$ for any set $N \subset E$.

Definition 2 A point function $\Phi$ is said to satisfy the Strong Lusin Condition $(S L C)$ on a set $E \subset[a, b]$ if for any set $N \subset E$ of measure zero and any $\epsilon>0$ there exists a positive function $\delta$ on $N$ such that $\sum_{j}\left|F\left(I_{j}\right)\right|<\epsilon$ for the associated function $F$ defined by (1) and for any $P \in \mathcal{P}(\delta, N)$.

The following Proposition is an obvious consequence of the definitions.

Proposition 1 A point function $\Phi$ satisfies $S L C$ on $E \subset[a, b]$ if and only if the associated interval function $F$ generates an absolutely continuous variational measure on $E$.

We need some definitions and results from the theory of the Denjoy-Perron integral. The reader is referred to [10] for the classical notion of $A C G_{*}$ functions. As for the notion of $V B_{*}$-functions and $V B G_{*}$-functions we are going to use here the following definitions which are equivalent to the classical ones. (See for example [11, pp. 77-79].)

Definition 3 An additive interval function $F$ is called a $V B_{*}$-function on a set $E \subset[a, b]$ if there exists a constant $M>0$ such that $\sum_{j}\left|F\left(I_{j}\right)\right|<M$ for any finite collection $\left\{I_{j}\right\}$ of nonoverlapping intervals with $I_{j}^{0} \cap E \neq \emptyset$.

Definition 4 An additive interval function $F$ is called a $V B G_{*}$-function on a set $E \subset[a, b]$ if $E=\cup_{k=1}^{\infty} E_{k}$ and $F$ is a $V B_{*}$-function on each $E_{k}$.

Remark 1 It is common to say that a point function $\Phi$ is an $A C G_{*}$-function or a $V B G_{*}$-function if the associated interval function $F$ defined by (1) is of the corresponding type.

A partial descriptive characterization of the $H K$-integral is given by the following known result. 
Proposition 2 ([2], [7], [8]) Let $F$ be an additive interval function that is differentiable a.e. in $[a, b]$. Then $F$ generates an absolutely continuous variational measure on $[a, b]$ if and only if $F^{\prime}$ is $H K$-integrable and $F$ is an indefinite $H K$-integral of $F^{\prime}$.

The following Proposition gives the known descriptive characterization of the Denjoy-Perron integral.

Proposition 3 ([10]) An additive interval function $F$ is an indefinite DenjoyPerron integral on $[a, b]$ if and only if $F$ is an $A C G_{*}$-function on $[a, b]$.

Proposition 4 ([1], [3], [4], [5]) The HK-integral is equivalent to the DenjoyPerron integral.

Proposition 5 ([10]) If an additive interval function is a $V B G_{*}$-function on a set $E$, then it is differentiable a.e. on $E$.

\section{Main Theorems}

Now we prove the principal technical result of this paper.

Theorem 1 If the variational measure $\mathrm{V}_{F}$ generated by an additive function $F$ is absolutely continuous on a closed set $E \subset[a, b]$, then $F$ is a $V B G_{*}$ function on $E$.

Proof. Suppose that $F$ is not a $V B G_{*}$-function on $E$. Let $P \subset E$ be the set of all the points $x \in E$ such that $F$ is not a $V B G_{*}$-function on $E \cap I$ for any interval $I, x \in I^{0}$. It is clear that $P$ is perfect and $F$ is not a $V B_{*}$-function on $P \cap I$ for any interval $I$ with $P \cap I^{0} \neq \emptyset$. We shall construct a set $N \subset P$ with $|N|=0$ and $V_{F}(N) \geq 1$ getting a contradiction to the assumption on the absolute continuity of $\mathrm{V}_{F}$.

As $F$ is not a $V B_{*}$-function on $P$ we can choose a finite collection of nonoverlapping closed intervals $\left\{I_{j}^{(1)}\right\}$ with $P \cap\left(I_{j}^{(1)}\right)^{0} \neq \emptyset$ for each $j$ such that $\sum_{j} \mid F\left(I_{j}^{(1)} \mid>1\right.$. We can suppose that the collection contains more than one interval and that $\sum_{j}\left|I_{j}^{(1)}\right|<2^{-1}$.

We proceed by induction. Having constructed a collection of intervals $\left\{I_{i}^{(k-1)}\right\}, k>0$, with $P \cap\left(I_{i}^{(k-1)}\right)^{0} \neq \emptyset$ for each $i$, we can construct a new finite collection of non-overlapping closed intervals $\left\{I_{j}^{(k)}\right\}$ such that

(i) $P \cap\left(I_{j}^{(k)}\right)^{0} \neq \emptyset$, 
(ii) each $I_{j}^{(k)}$ is contained in some $I_{i}^{(k-1)}$,

(iii) each $I_{i}^{(k-1)}$ contains at least two intervals $I_{j}^{(k)}$,

(iv) $\sum_{j}\left|I_{j}^{(k)}\right|<2^{-k}$,

(v) $\sum_{j: I_{j}^{(k)} \subset I_{i}^{(k-1)}}\left|F\left(I_{j}^{(k)}\right)\right|>1$ for each $i$.

We put $N=\cap_{k} \cup_{j} I_{j}^{(k)}$. Because of (i)-(iv) the set $N$ is perfect, $N \subset P$ and $|N|=0$. Take any positive function $\delta(x)$ defined on $N$ and let

$$
N_{n}=\left\{x \in N: \delta(x)>n^{-1}\right\} .
$$

By Baire category theorem, $N_{n}$ for some $n$ is dense in some portion of $N$ defined by some interval $J$. We can find $l$ and $i$ such that $I_{i}^{(l)} \subset J,\left|I_{i}^{(l)}\right|<n^{-1}$ and so the family $\left(I_{j}^{(l+1)}, x_{j}\right)$ with $x_{j} \in I_{j}^{(l+1)} \cap N_{n}, I_{j}^{(l+1)} \subset I_{i}^{(l)}$ is a $\delta$-fine partition anchored in $N$. In view of (v) with $k$ replaced by $l+1$ we get

$$
\operatorname{Var}(F, \delta, N) \geq \sum_{j: I_{j}^{(l+1)} \subset I_{i}^{(l)}}\left|F\left(I_{j}^{(l+1)}\right)\right|>1
$$

and therefore $\mathrm{V}_{F}(N) \geq 1$ giving a desired contradiction.

Theorem 1 combined with Proposition 5 gives the following result.

Theorem 2 If the variational measure generated by an additive function $F$ is absolutely continuous on $[a, b]$, then $F$ is differentiable a.e. on $[a, b]$.

In view of Proposition 1 we can reformulate this result in terms of SLC.

Corollary 1 If a point function $\Phi$ satisfies SLC on $[a, b]$, then $\Phi$ is differentiable a.e. on $[a, b]$.

Now Theorem 2 together with Proposition 2 shows that the absolute continuity of $\mathrm{V}_{F}$ is a full descriptive characterization of the $H K$-integral. Namely the following proposition holds.

Theorem 3 An additive function $F$ is an indefinite $H K$-integral of some function defined on $[a, b]$ if and only if $F$ generates an absolutely continuous variational measure. In other words, the class of all indefinite $\mathrm{HK}$-integrals coincides with the class of all additive functions generating absolutely continuous variational measures. 
It is nice that this descriptive characterization of the $H K$-integral looks very similar to the classical characterization of the Lebesgue integral as an absolutely continuous set function.

Proposition 3, Proposition 4 and Theorem 3 imply the following assertion.

Theorem 4 The class of all $A C G_{*}$-functions coincides with the class of all additive functions $F$ generating absolutely continuous variational measure (or for point functions: with the class of all functions satisfying SLC).

\section{Pfeffer's Problem}

Now we turn to W. Pfeffer's question on $\sigma$-finiteness of a variational measure. This question in [9] refers to a slightly different variational measure defined by nonnegative functions $\delta$ instead of positive ones. So we introduce some changes into the definitions of Section 2.

A nonnegative function $\delta$ is called a gage on $E \subset[a, b]$ whenever its null set $Z=\{x \in E: \delta(x)=0\}$ is countable. (In the multidimensional case it should be "thin" in a certain sense (see [8]), but in the one-dimensional case we are concerned here with, "thin" means countable.)

The definition of $\delta$-fine partition in the case of a gage instead of a positive function coincides with one given in Section 2 and for the $\delta$-variation defined by these kind of partitions we keep the notation given by formula (2).

If in the definition given by formula (3) "inf" is taken over all gages defined on $E$, we denote by $\mathrm{V}_{F}^{g}$ the resulting gage-variational measure generated by $F$.

In these terms a special case of Pfeffer's question we are considering here is: If $\mathrm{V}_{F}^{g}$ is absolutely continuous on a set (in the sense of the Definition 1), is it $\sigma$-finite on this set?

We show that the positive answer to this question follows from the results of Section 3. We start with proving that in the one-dimensional case the gagevariational measure $\mathrm{V}_{F}^{g}$ coincides with the variational measure $\mathrm{V}_{F}$ defined by positive functions $\delta$.

Proposition 6 Let $F$ be an additive continuous function on $[a, b]$. Then for each $E \subset[a, b]$, we have $\mathrm{V}_{F}^{g}(E)=\mathrm{V}_{F}(E)$.

Proof. We follow the proof given in $[8, \mathrm{p} .117]$ to a proposition which is in fact a particular case of the present Proposition.

Let $E$ be a fixed set on $[a, b]$. Since each positive gage is a gage, $\mathrm{V}_{F}^{g}(E) \leq$ $\mathrm{V}_{F}(E)$. So we are to prove that $\mathrm{V}_{F}^{g}(E) \geq \mathrm{V}_{F}(E)$. To this end take any $\varepsilon>0$, and any gage $\delta^{*}$ on $E$. Let $\left\{z_{1}, z_{2}, \ldots\right\}$ be an enumeration of the set $Z=\left\{x \in E: \delta^{*}(x)=0\right\}$. As $F$ is continuous, there are $\gamma_{n}>0$ such that 
$|F(I)|<\varepsilon 2^{-n}$, for each interval $I \subset[a, b] \cap\left[z_{n}-\gamma_{n}, z_{n}+\gamma_{n}\right]$. Now define a positive function $\delta$ on $E$ by setting

$$
\delta(x)= \begin{cases}\delta^{*}(x), & \text { if } x \in E \backslash Z, \\ \gamma_{n}, & \text { if } x=z_{n} \text { and } n=1,2, \ldots,\end{cases}
$$

and choose a $\delta$-fine partition $\left\{\left(I_{1}, x_{1}\right), \ldots,\left(I_{p}, x_{p}\right)\right\}$ in $[a, b]$ anchored in $E$ such that

$$
\operatorname{Var}(F, \delta, E) \leq \sum_{i=1}^{p}\left|F\left(I_{i}\right)\right|+\varepsilon
$$

By choice of $\gamma_{n}$ we have $\left|F\left(I_{i}\right)\right|<\varepsilon 2^{-n}$ if $x_{i}=z_{n}$. Thus we obtain

$$
\begin{aligned}
\sum_{i=1}^{p}\left|F\left(I_{i}\right)\right| & \leq \sum_{i: x_{i} \notin Z}\left|F\left(I_{i}\right)\right|+\sum_{i: x_{i} \in Z}\left|F\left(I_{i}\right)\right| \\
& <\operatorname{Var}\left(F, \delta^{*}, E\right)+\sum_{n=1}^{\infty} \varepsilon 2^{-n}=\operatorname{Var}\left(F, \delta^{*}, E\right)+\varepsilon
\end{aligned}
$$

This inequality combined with (4) and (3) implies

$$
\mathrm{V}_{F}(E) \leq \operatorname{Var}(F, \delta, E)<\operatorname{Var}\left(F, \delta^{*}, E\right)+2 \varepsilon
$$

Since $\varepsilon$ is arbitrary and $\delta^{*}$ does not depend on $\varepsilon$, we have

$$
\mathrm{V}_{F}(E) \leq \operatorname{Var}\left(F, \delta^{*}, E\right)
$$

Now taking "inf" over all gages $\delta^{*}$ on $E$ we finally get $\mathrm{V}_{F}(E) \leq \mathrm{V}_{F}^{g}(E)$.

So the question related to $\mathrm{V}_{F}^{g}$ is reduced to the one related to $\mathrm{V}_{F}$. But for this variational measure the following result is known (see [11, p. 79]).

Proposition 7 The variational measure generated by an additive function $F$ is $\sigma$-finite on a Borel set $E \subset[a, b]$ if and only if $F$ is a $V B G_{*}$-function on $E$.

Therefore from Theorem 1 and Proposition 6 we get the following.

Theorem 5 If the gage-variational measure $\mathrm{V}_{F}^{g}$ generated by an additive continuous function $F$ is absolutely continuous on a closed set $E \subset[a, b]$, then it is $\sigma$-finite on $E$. 
Note that in the multidimensional case and in the case of partitions by figures instead of intervals the present proof does not work not only because we do not have (for the multidimensional case) an analogue to the Proposition 6, but also because a new parameter of regularity of partitions involved in the definition of the variational measure in these cases prevents a direct application of the scheme used here in the proof of Theorem 1. But if the variational measure is defined by positive gages and by partitions with a fixed parameter of regularity, then the analogues of Theorems 1 and 5 in the multidimensional case can be proved essentially in the same way.

\section{References}

[1] R. Gordon, A descriptive characterization of the generalized Riemann integral, Real Analysis Exch., 15 (1) (1989-90), 397-400.

[2] J. Jarnik and J. Kurzweil, A general form of the product integral and linear ordinary differential equations, Czech. Math. J., 37 (112) 1987, 642-659.

[3] J. Kurzweil, Nichtabsolut konvergente Integrale, Teubner-Texte zur Mathematik, 26, Teubner, Leipzig, 1980.

[4] R. Henstock, General theory of integration, Clarendon Press, Oxford, 1991.

[5] P. Y. Lee, Lanzhou lectures on Henstock integration, World Scientific, Singapore, 1989.

[6] P. Y. Lee, On ACG* functions, Real Analysis Exch., 15 (1989-90), 754759 .

[7] P. Y. Lee and R. Vyborny, Kurzweil-Henstock integration and strong Lusin condition, Boll. U.M.I. (7), 7-B (1993), 761-773.

[8] W. F. Pfeffer, The Riemann Approach to Integration, Cambridge Univ. Press, Cambridge, 1993.

[9] W. F. Pfeffer, On additive continuous functions of figures, to appear.

[10] S. Saks, Theory of the integral, Dover, New York, 1964.

[11] B. S. Thomson, Derivates of intervals functions, Memoir Amer. Math. Soc., 452, Providence, 1991. 